\title{
Asymptotic analysis for transient scattered field excited by the edges of a cylindrically curved conducting open sheet
}

\author{
Keiji Goto $^{\text {a) }}$, Mizuki Sawada, Kojiro Mori, and Yuki Horii \\ Department of Communication Engineering, National Defense Academy, \\ 1-10-20 Hashirimizu, Yokosuka, Kanagawa 239-8686, Japan \\ a)keigoto@nda.ac.jp
}

Abstract: Asymptotic analysis for the transient scattered field when a pulse wave is incident on the edges of a cylindrically curved conducting open sheet is presented. By applying only the Fourier transform method, we derive a novel time-domain (TD) asymptotic solution. The TD asymptotic solution is represented by a superposition of the transient scattered filed elements arrived at the observation point. The validity of the TD asymptotic solution proposed here is confirmed by comparing with both the reference solution and the experimental-numerical results. We show the interesting phenomenon that the creeping wave propagates along the curved conducting sheet later than the whispering-gallery mode.

Keywords: asymptotic analysis, transient scattered field, cylindrically curved conducting open sheet, time-domain, asymptotic solution

Classification: Electromagnetic theory

\section{References}

[1] A. Michaeli: IEEE Trans. Antennas Propag. 37 (1989) 1073.

[2] P. R. Rousseau and P. H. Pathak: IEEE Trans. Antennas Propag. 43 (1995) 1375.

[3] F. A. Molinet: IEEE Antennas Propag. Mag. 47 (2005) 34.

[4] K. Goto, T. Kawano and T. Ishihara: IEICE Trans. Electron. E92-C (2009) 25.

[5] K. Goto, T. Kawano and T. Ishihara: IEICE Electron. Express 5 (2008) 983.

[6] K. Goto, T. Kawano and T. Ishihara: IEICE Electron. Express 6 (2009) 354 .

[7] K. Goto and L. H. Loc: The Papers of Technical Meeting on Electromagnetic Theory, IEE Japan EMT-12-75 [2] (2012) 61.

[8] K. Goto, T. Ajiki, T. Kawano and T. Ishihara: ISAP (2006) 2D2b2.

[9] M. Abramowitz and I. A. Stegun: Handbook of mathematical Functions (Dover, New York, 1972) 358. 


\section{Introduction}

By recent technological advances in the area of radar cross section, high resolution radar, and target identification, it is becoming important to study the asymptotic analysis methods for the frequency-domain (FD) and the time-domain (TD) scattered fields by various kinds of curved structures $[1,2,3,4,5,6]$.

In the previous study [6], we have considered the asymptotic analyses for the whispering-gallery mode radiation field (WG), the geometric optical ray (GO), the surface diffracted ray (SD) converted from the creeping wave $(\mathrm{CW})$, the edge diffracted ray (ED), and the edge diffracted and reflected ray (EDR) which form the transient scattered field by a cylindrically curved conducting surface. We have derived the TD asymptotic solution for the transient scattered field by applying the saddle point technique [5] to the WG and applying the Fourier transform method [6] to the GO, the $\mathrm{SD}$, the ED, and the EDR. However, in order to derive the abovementioned TD asymptotic solution, it is necessary to use two kinds of asymptotic analysis methods. For this reason, it has the problem that the derivation process of the TD asymptotic solution is complicated.

In the present study, we challenge again about the asymptotic analysis for the transient scattered field when a pulse wave is incident on the edges of a cylindrically curved conducting open sheet. By applying only the Fourier transform method, we derive a novel TD asymptotic solution by a combination of the WG, the $\mathrm{SD}$, the $\mathrm{CW}$ incidence and edge diffracted ray (CWED), the reflected GO (RGO), and the reflected and edge diffracted ray (RED) $[1,4]$. The validity of the TD asymptotic solution derived by applying the unified asymptotic analysis method is confirmed by comparing with both the reference solution and the experimental-numerical results. We show the interesting phenomenon that the $\mathrm{CW}$ propagates along the curved conducting sheet later than the whispering-galley mode. The time factor $\exp (-i \omega t)$ is adopted and suppressed here.

\section{Formulation and frequency-domain (FD) asymptotic solu- tion}

Fig. 1 (a) shows the cylindrically curved conducting open sheet with edges $\mathrm{A}$ and $\mathrm{B}$ defined by $\rho=a, 0 \leq \phi \leq \phi_{\mathrm{AB}}$, and the coordinate systems $(x, y, z),(\rho, \phi)$, and $(r, \psi)$. We assume that the radius of curvature $a$ is sufficiently large compared with a wavelength $\lambda$ (i.e., $\omega a / c>>1, \omega$ : angular frequency, $c$ : speed of light) and that the plane wave which has a magnetic field component in the $z$-direction propagates the positive $x$-direction. We have shown in Fig. 1 (b) the loci or the propagation paths of the $m$ th-order WG $\left(\mathrm{WG}_{m}\right)$ (1) and (2), the $n$ th-order SD $\left(\mathrm{SD}_{n}\right)(\mathbf{3})$, the $n$ th-order CWED $\left(\mathrm{CW}_{n} \mathrm{ED}\right)(\mathbf{4})$, the $k$ times reflected $\mathrm{GO}\left(\mathrm{RGO}_{k}\right)(\mathbf{5}$ $(k=0)$ and $6(k=1))$, and the $k$ times reflected and edge diffracted ray $\left(\operatorname{RED}_{k}\right)(\mathbf{8}(k=0)$ and $8(k=1))$ excited by the equivalent source at the edge A after the magnetic type (H-type) plane wave is incident on the edge A.

The propagation phenomena of the scattered field when the H-type plane wave is incident on the other edge B are closely similar to those of the scattered field shown in Fig. 1 (b) (see Figs. 3 (a) and 3 (b)). So, we 


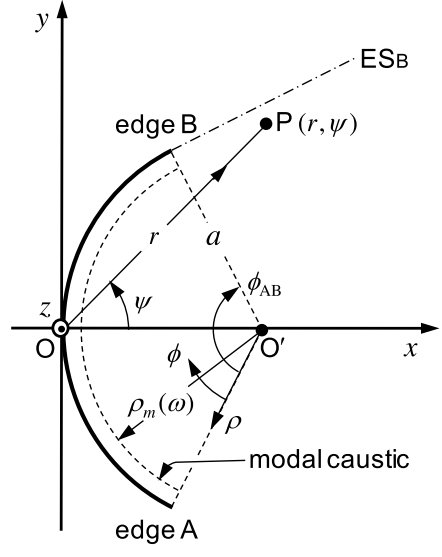

(a) Curved sheet $\left(a, \phi_{\mathrm{AB}}\right)$ and coordinate systems $(x, y, z)$, $(\rho, \phi)$, and $(r, \psi)$.

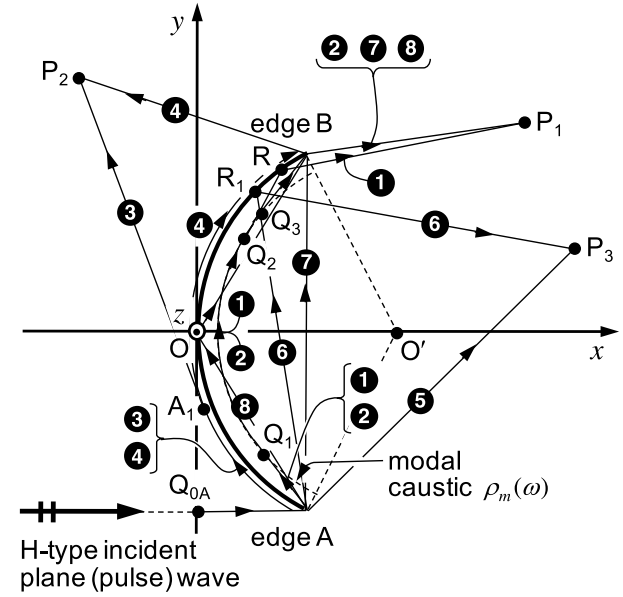

(b) Propagation paths of scattered field elements excited by the edge A.

Fig. 1. Propagation phenomena of scattered field excited by the edge $\mathrm{A}$ of a cylindrically curved conducting open sheet $\left(a, \phi_{\mathrm{AB}}\right)$. $\mathrm{ES}_{\mathrm{B}}$ : tangent to the edge $\mathrm{B}$, $\rho_{m}(\omega)$ : modal caustic of $m$ th-order whisperinggallery mode.

consider only the scattering problem for the incident plane wave on the edge $\mathrm{A}$ of the curved sheet.

The $z$-component of the scattered magnetic field observed at the point $\mathrm{P}(r, \psi)$ after excited by the edge $\mathrm{A}$ may be represented as follows $[1,4,6,8]$ :

$$
u_{\mathrm{A}}(\omega) \sim \exp \left(i \omega L_{\mathrm{Q}_{0 \mathrm{~A}} \mathrm{~A}} / c\right) \sum_{i}\left[\sum_{r}^{R} U_{i}(\mathrm{P}) u_{i}(\omega)\right],
$$

where $(i, r, R)=\left(\mathrm{WG}_{m}, m=1, M\right),\left(\mathrm{SD}_{n}, n=1, N_{1}\right)$,

$$
\begin{aligned}
& \left(\mathrm{CW}_{n} \mathrm{ED}, n=1, N_{2}\right),\left(\mathrm{RGO}_{k}, k=0, K_{1}\right), \\
& \left(\mathrm{RED}_{k}, k=0, K_{2}\right) .
\end{aligned}
$$

Here $L_{\mathrm{Q}_{0 \mathrm{~A}} \mathrm{~A}}$ is the propagation distance along the path $\mathrm{Q}_{0 \mathrm{~A}} \rightarrow \mathrm{A}$ of the $\mathrm{H}$ type incident plane wave which illuminates the edge $\mathrm{A}$ from the reference point $\mathrm{Q}_{0 \mathrm{~A}}$ on the $y$-axis (see Fig. $1(\mathrm{~b})$ ). While $u_{\mathrm{WG}_{m}}(\omega), u_{\mathrm{SD}_{n}}(\omega)$, $u_{\mathrm{CW}_{n} \mathrm{ED}}(\omega), u_{\mathrm{RGO}_{k}}(\omega)$, and $u_{\mathrm{RED}_{k}}(\omega)$ denote respectively the FD asymptotic solutions for the $\mathrm{WG}_{m}$, the $\mathrm{SD}_{n}$, the $\mathrm{CW}_{n} \mathrm{ED}$, the $\mathrm{RGO}_{k}$, and the $\mathrm{RED}_{k}$. The function $U_{i}(\mathrm{P})$ is defined as $U_{i}(\mathrm{P})=1$ (or $U_{i}(\mathrm{P})=0$ ) when the scattered field element $u_{i}(\omega)$ can (or cannot) reach the point $\mathrm{P}$.

Please note that in order to avoid the confusion of the propagation paths of the scattered field elements arriving at the point $\mathrm{P}$, we examine the FD asymptotic solutions for every few scattered filed elements which arrive at the point $\mathrm{P}_{1}, \mathrm{P}_{2}$, or $\mathrm{P}_{3}$ depicted in Fig. 1 (b) from now on.

First, the FD asymptotic solution $u_{\mathrm{WG}_{m}}(\omega)$ for the $\mathrm{WG}_{m}$ arrived at the point $\mathrm{P}_{1}$ in Fig. 1 (b) may be given by a combination of the GO $\left(u_{m, \mathrm{GO}}(\omega)\right)$ and the $\mathrm{ED}\left(u_{m, \mathrm{ED}}(\omega)\right)$ converted from the $m$ th-order WG mode $[4,5,7,8]$.

$$
\begin{aligned}
& u_{\mathrm{WG}_{m}}(\omega)=U_{\mathrm{WG}_{m}}\left(\mathrm{P}_{1}\right) u_{m, \mathrm{GO}}(\omega)+u_{m, \mathrm{ED}}(\omega), \\
& U_{\mathrm{WG}_{m}}\left(\mathrm{P}_{1}\right)=1 \text { for lit region, } U_{\mathrm{WG}_{m}}\left(\mathrm{P}_{1}\right)=0 \text { for shadow region, }
\end{aligned}
$$




$$
\begin{gathered}
u_{m, \mathrm{GO}}(\omega)=A_{m, \mathrm{GO}}(\omega) \exp \left(i \omega L_{m, \mathrm{GO}}(\omega) / c\right), \\
u_{m, \mathrm{ED}}(\omega)=A_{m, \mathrm{ED}}(\omega) \exp \left[i \omega\left(L_{m, \mathrm{ED}}(\omega) / c+L_{\mathrm{BP}_{1}} / c\right)\right] .
\end{gathered}
$$

Where $A_{m, \mathrm{GO}}(\omega)\left(A_{m, \mathrm{ED}}(\omega)\right)$ is the amplitude term of the GO (ED). Notation $L_{m, \mathrm{GO}}(\omega)$ in $(3)$ denotes the propagation distance along the path of the GO converted from the modal ray $\left(\mathrm{A} \rightarrow \mathrm{Q}_{1} \cup \mathrm{Q}_{2} \rightarrow \mathrm{R} \rightarrow \mathrm{P}_{1}\right)$. Here $Q_{1} \cup \mathrm{Q}_{2}$ has been used to indicated the WG mode propagation from the point $\mathrm{Q}_{1}$ to the point $\mathrm{Q}_{2}$ along the arc of the $m$ th-order modal caustic $\rho=\rho_{m}(\omega)$ which changes as the function of the angular frequency $\omega$ (see Figs. 1 (a) and 1 (b)) $[4,6,7,8]$. While, $L_{m, \mathrm{ED}}(\omega)$ and $L_{\mathrm{BP}_{1}}$ in (4) denote respectively the propagation distance along the path of the modal ray $\left(\mathrm{A} \rightarrow \mathrm{Q}_{1} \cup \mathrm{Q}_{3} \rightarrow \mathrm{B}\right)$ which propagates from the edge $\mathrm{A}$ to the other edge $\mathrm{B}$ and that of the cylindrical wave $\left(\mathrm{B} \rightarrow \mathrm{P}_{1}\right)$ which reaches the point $\mathrm{P}_{1}$ after diffracted by the edge B. Note that the propagation distances $L_{m, \mathrm{GO}}(\omega)$ and $L_{m, \mathrm{ED}}(\omega)$ change as the function of $\omega$.

Second, the FD asymptotic solution $u_{\mathrm{SD}_{n}}(\omega)$ for the $\mathrm{SD}_{n}$ with the path $\mathrm{A} \curvearrowright \mathrm{A}_{1} \rightarrow \mathrm{P}_{2}$ arrived at the point $\mathrm{P}_{2}$ shown in Fig. 1 (b) and the FD asymptotic solution $u_{\mathrm{CW}_{n} \mathrm{ED}}(\omega)$ for the $\mathrm{CW}_{n} \mathrm{ED}$ with the path $\mathrm{A} \curvearrowright \mathrm{B} \rightarrow \mathrm{P}_{2}$ may be expressed by $[1,4,6,7,8]$ :

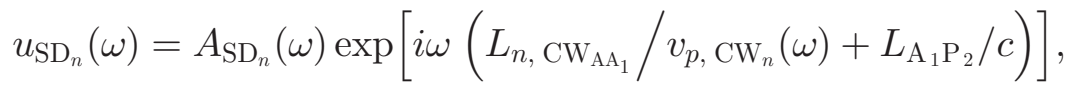

$$
\begin{aligned}
& u_{\mathrm{CW}_{n} \mathrm{ED}}(\omega)=A_{\mathrm{CW}_{n} \mathrm{ED}}(\omega) \exp \left[i \omega\left(L_{n}, \mathrm{CW}_{\mathrm{AB}} / v_{p}, \mathrm{CW}_{n}(\omega)+L_{\mathrm{BP}_{2}} / c\right)\right] .
\end{aligned}
$$

Here $A_{\mathrm{SD}_{n}}(\omega)\left(A_{\mathrm{CW}_{n} \mathrm{ED}}(\omega)\right)$ is the amplitude term of the $\mathrm{SD}_{n}\left(\mathrm{CW}_{n} \mathrm{ED}\right)$. Notation $L_{n}, \mathrm{CW}_{\mathrm{AA}_{1}}\left(L_{n}, \mathrm{CW}_{\mathrm{AB}}\right)$ denotes the propagation distance along the path of the $n$ th-order $\mathrm{CW}\left(\mathrm{CW}_{n}\right)\left(\mathrm{A} \curvearrowright \mathrm{A}_{1}(\mathrm{~A} \curvearrowright \mathrm{B})\right)$ which is excited by the edge $\mathrm{A}$ and propagates along the convex surface $\rho=a$ to the surface diffraction point $\mathrm{A}_{1}$ (the edge $\mathrm{B}$ ). Also $L_{\mathrm{A}_{1} \mathrm{P}_{2}}\left(L_{\mathrm{BP}_{2}}\right)$ is the propagation distance of the cylindrical wave $\left(\mathrm{A}_{1} \rightarrow \mathrm{P}_{2}\left(\mathrm{~B} \rightarrow \mathrm{P}_{2}\right)\right)$ which propagates from the point $\mathrm{A}_{1}(\mathrm{~B})$ to the point $\mathrm{P}_{2}$. While, $v_{p, \mathrm{CW}_{n}}(\omega)$ in (5) and (6) denotes the phase velocity of the $\mathrm{CW}_{n}$ defined by

$$
\begin{gathered}
v_{p, \mathrm{CW}_{n}}(\omega)=c / c_{n}(\omega)(<c), \\
c_{n}(\omega)=1+\left(\sigma_{n} / 4\right)((2 c) /(\omega a))^{2 / 3}(>1),
\end{gathered}
$$

where $\sigma_{n}$ is the $n$th zero of the Airy function derivative (i.e., $A i^{\prime}\left(\sigma_{n}\right)=0$ ) [9].

Finally, the FD asymptotic solution $u_{\mathrm{RGO}_{k}}(\omega)$ for the $\mathrm{RGO}_{k}$ with the path $\mathrm{A} \rightarrow \mathrm{P}_{3}(k=0)$ or $\mathrm{A} \rightarrow \mathrm{R}_{1} \rightarrow \mathrm{P}_{3}(k=1)$ arrived at the point $\mathrm{P}_{3}$ in Fig. 1 (b) may be given by $[4,6,7,8]$ :

$$
u_{\mathrm{RGO}_{k}}(\omega)=A_{\mathrm{RGO}_{k}}(\omega) \exp \left(i \omega L_{\mathrm{RGO}_{k}} / c\right) .
$$

Notation $A_{\mathrm{RGO}_{k}}(\omega)$ is the amplitude term of the $\mathrm{RGO}_{k}$ and $L_{\mathrm{RGO}_{k}}$ denotes the propagation distance along the path $\mathrm{A} \rightarrow \mathrm{P}_{3}(k=0)$ or $\mathrm{A} \rightarrow \mathrm{R}_{1} \rightarrow \mathrm{P}_{3}(k=1)$ which is excited by the edge $\mathrm{A}$ and reaches the point $\mathrm{P}_{3}$ directly $(k=0)$ or reaches the point $\mathrm{P}_{3}$ after reflected at the point $\mathrm{R}_{1}$ on the concave sheet $(k=1)$. By the same manner, the FD asymptotic solution $u_{\mathrm{RED}_{k}}(\omega)$ for the $\mathrm{RED}_{k}$ with the path $\mathrm{A} \rightarrow \mathrm{B} \rightarrow \mathrm{P}_{1}(k=0)$ or $\mathrm{A} \rightarrow \mathrm{O} \rightarrow \mathrm{B} \rightarrow \mathrm{P}_{1}(k=1)$ arrived at the point $\mathrm{P}_{1}$ shown in Fig. 1 (b) may be given by $[4,6]$ : 


$$
u_{\mathrm{RED}_{k}}(\omega)=A_{\mathrm{RED}_{k}}(\omega) \exp \left(i \omega L_{\mathrm{RED}_{k}} / c\right) .
$$

Here $A_{\mathrm{RED}_{k}}(\omega)$ and $L_{\mathrm{RED}_{k}}$ denote respectively the amplitude term and the propagation distance of the $\mathrm{RED}_{k}$.

\section{Time-domain (TD) asymptotic solution}

The transient scattered field $y_{\mathrm{A}}(t)$ arrived at the observation point $\mathrm{P}(r, \psi)$ after excited by the edge A may be represented by the inverse Fourier transform of the product of the FD scattered filed $u_{\mathrm{A}}(\omega)$ and the frequency spectrum $S(\omega)$ of a pulse source function $s(t)[5,6,7,8]$.

$$
\begin{aligned}
y_{\mathrm{A}}(t) & =\frac{1}{2 \pi} \int_{-\infty}^{\infty} u_{\mathrm{A}}(\omega) S(\omega) \exp (-i \omega t) d \omega \\
& =\sum_{i}\left[\sum_{r}^{R} U_{i}(\mathrm{P}) y_{i}(t)\right],
\end{aligned}
$$

where $(i, r, R)=\left(\mathrm{WG}_{m}, m=1, M\right),\left(\mathrm{SD}_{n}, n=1, N_{1}\right)$, $\left(\mathrm{CW}_{n} \mathrm{ED}, n=1, N_{2}\right),\left(\mathrm{RGO}_{k}, k=0, K_{1}\right)$, $\left(\mathrm{RED}_{k}, k=0, K_{2}\right)$.

$$
y_{i}(t)=\frac{1}{2 \pi} \int_{-\infty}^{\infty} \exp \left(i \omega L_{\mathrm{Q}_{0} \mathrm{~A}} / c\right) u_{i}(\omega) S(\omega) \exp (-i \omega t) d \omega .
$$

We assume the following truncated pulse source $s(t)$ defined by the product of the modulated wave $s_{0}(t)$ and the carrier wave $\exp \left[-i \omega_{0}\left(t-t_{0}\right)\right]$ whose central angular frequency is $\omega_{0}$ :

$$
\begin{aligned}
& s(t)=s_{0}(t) \exp \left[-i \omega_{0}\left(t-t_{0}\right)\right] \text { for } 0 \leq t \leq 2 t_{0}, \\
& s(t)=0 \quad \text { for } t<0, t>2 t_{0},
\end{aligned}
$$

where $t_{0}$ denotes the constant parameter. Denoting the Fourier transform of $s_{0}(t)$ by $S_{0}(\omega)$, the Fourier transform $S(\omega)$ of $s(t)$ can be represented by

$$
S(\omega)=S_{0}\left(\omega-\omega_{0}\right) \exp \left(i \omega_{0} t_{0}\right) .
$$

\subsection{TD asymptotic solution for transient $\mathbf{W G}_{\boldsymbol{m}}$}

Substituting $u_{\mathrm{WG}_{m}}(\omega)$ in (2) associated with (3) and (4) into the $y_{\mathrm{WG}_{m}}(t)$ in (11) yields the integral representation for the transient $\mathrm{WG}_{m}$.

$$
\begin{gathered}
y_{\mathrm{WG}_{m}}(t)=U(\mathrm{P}) y_{m, \mathrm{GO}}(t)+y_{m, \mathrm{ED}}(t), U(\mathrm{P})=\left\{\begin{array}{l}
1: \text { lit region } \\
0: \text { shadow region }
\end{array}\right. \\
y_{\mathrm{m}, j}(t)=\frac{\exp \left(i \omega_{0} t_{0}\right)}{2 \pi} \int_{-\infty}^{\infty} A_{m, j}(\omega) S_{0}\left(\omega-\omega_{0}\right) \exp \left[-i\left\{\omega t-h_{m, j}(\omega)\right\}\right] d \omega \\
h_{m, j}(\omega)=\omega\left(L / c+L_{m, j}(\omega) / c\right), \quad L= \begin{cases}L_{\mathrm{Q}_{0 \mathrm{~A}} \mathrm{~A}}, j \equiv \mathrm{GO} \\
L_{\mathrm{Q}_{0 \mathrm{~A}} \mathrm{~A}}+L_{\mathrm{BP}_{1}}, j \equiv \mathrm{ED}\end{cases}
\end{gathered}
$$

In the integral $y_{m, j}(t), j \equiv \mathrm{GO}$ or $j \equiv \mathrm{ED}$, in (15) associated with (16), we assume that the functions $A_{m, j}(\omega)$ and $h_{m, j}(\omega)$ in the integrand may be approximated near the central angular frequency $\omega=\omega_{0}$ by 


$$
A_{m, j}(\omega) \sim A_{m, j}\left(\omega_{0}\right), \quad h_{m, j}(\omega) \sim h_{m, j}\left(\omega_{0}\right)+\left(\omega-\omega_{0}\right) h_{m, j}^{\prime}\left(\omega_{0}\right) .
$$

Substituting the approximations in (17) into (15), the integral in (15) is reduced to

$$
\begin{gathered}
y_{m, j}(t) \sim A_{m, j}\left(\omega_{0}\right) \exp \left[-i \omega_{0}\left(t-t_{0}-L / c-L_{m, j}\left(\omega_{0}\right) / c\right)\right] I_{m, j}(t), \\
I_{m, j}(t)=\frac{1}{2 \pi} \int_{-\infty}^{\infty} S_{0}\left(\omega-\omega_{0}\right) \exp \left[-i\left(\omega-\omega_{0}\right)\left(t-L / c-L_{m, j}\left(\omega_{0}\right) / v_{g, m, j}\right)\right] d \omega .
\end{gathered}
$$

The integral $I_{m, j}(t)$ in (19) is represented by the inverse Fourier transform of $S_{0}\left(\omega-\omega_{0}\right)$. Then utilizing the Fourier transform method $[6,7,8], I_{m, j}(t)$ may be represented by

$$
I_{m, j}(t)=s_{0}\left(t-t_{p, m, j}\right), \quad t_{p, m, j}=L / c+L_{m, j}\left(\omega_{0}\right) / v_{g, m, j} .
$$

By substituting (20) into (18), one may obtain the TD asymptotic solution for the transient GO and the transient ED which form the transient $\mathrm{WG}_{m}$ :

$$
y_{m, j}(t) \sim A_{m, j}\left(\omega_{0}\right) s_{0}\left(t-t_{p, m, j}\right) \exp \left[-i \omega_{0}\left(t-t_{0}-L / c-L_{m, j}\left(\omega_{0}\right) / c\right)\right] .
$$

Here the group velocity of the GO element along the path $\mathrm{A} \rightarrow \mathrm{Q}_{1} \cup \mathrm{Q}_{2} \rightarrow \mathrm{R} \rightarrow \mathrm{P}_{1}\left(\equiv L_{m, \mathrm{GO}}\left(\omega_{0}\right)\right)$ and the modal ray element along the path $\left(\mathrm{A} \rightarrow \mathrm{Q}_{1} \cup \mathrm{Q}_{3} \rightarrow \mathrm{B}\right)\left(\equiv L_{m, \mathrm{ED}}\left(\omega_{0}\right)\right)$ is defined by

$$
v_{g, m, j}=\frac{c}{1+\omega_{0} L_{m, j}^{\prime}\left(\omega_{0}\right) / L_{m, j}\left(\omega_{0}\right)}(<c), \quad L_{m, j}^{\prime}\left(\omega_{0}\right)(>0) .
$$

\subsection{TD asymptotic solutions for transient $\mathrm{SD}_{n}$ and transient $\mathrm{CW}_{n} \mathrm{ED}$}

Substituting $u_{\mathrm{SD}_{n}}(\omega)$ in (5) into the inverse Fourier transform $y_{\mathrm{SD}_{n}}(t)$ in (11) yields the integral representation for the transient $\mathrm{SD}_{n}$.

$$
\begin{gathered}
y_{\mathrm{SD}_{n}}(t)=\frac{\exp \left(-\omega_{0} t_{0}\right)}{2 \pi} \int_{-\infty}^{\infty} A_{\mathrm{SD}_{\mathrm{n}}}(\omega) S_{0}\left(\omega-\omega_{0}\right) \exp \left[-i\left\{\omega t-h_{\mathrm{SD}_{n}}(\omega)\right\}\right] d \omega, \\
h_{\mathrm{SD}_{n}}(\omega)=\omega\left(L_{\mathrm{Q}_{0 \mathrm{~A}} \mathrm{~A}} / c+L_{n}, \mathrm{CW}_{\mathrm{AA}_{1}} / v_{p, \mathrm{CW}_{n}}(\omega)+L_{\mathrm{A}_{1} \mathrm{P}_{2}} / c\right) .
\end{gathered}
$$

The integral $y_{\mathrm{SD}_{n}}(t)$ in (23) is described by the representation similar to the integral $y_{m, \mathrm{ED}}(t)$ in $(15)$.

By substituting the transformations for

$$
A_{m, \mathrm{ED}}(\omega) \rightarrow A_{\mathrm{SD}_{n}}(\omega), \quad L_{\mathrm{BP}_{1}} \rightarrow L_{\mathrm{A}_{1} \mathrm{P}_{2}}
$$

$L_{m, \mathrm{ED}}(\omega) / c \rightarrow \hat{L}_{n, \mathrm{CW}_{\mathrm{AA}_{1}}}(\omega) / c, \quad$ where $\hat{L}_{n, \mathrm{CW}_{\mathrm{AA}_{1}}}(\omega)=L_{n, \mathrm{CW}_{\mathrm{AA}_{1}}} c_{n}(\omega)$,

into the integral $y_{m, \mathrm{ED}}(t)$ in (15) associated with (16), and then applying the Fourier transform method in Section 3.1, one may derive the TD asymptotic solution for the transient $\mathrm{SD}_{n}$ :

$$
\begin{aligned}
y_{\mathrm{SD}_{n}}(t) \sim & A_{\mathrm{SD}_{n}}\left(\omega_{0}\right) s_{0}\left(t-t_{p, \mathrm{SD} n}\right) \\
& \cdot \exp \left[-i \omega_{0}\left(t-t_{0}-L_{\mathrm{Q}_{0 \mathrm{~A}} \mathrm{~A}} / c-L_{n}, \mathrm{CW}_{\mathrm{AA}_{1}} / v_{p, \mathrm{CW}_{n}}\left(\omega_{0}\right)-L_{\mathrm{A}_{1} \mathrm{P}_{2}} / c\right)\right],
\end{aligned}
$$




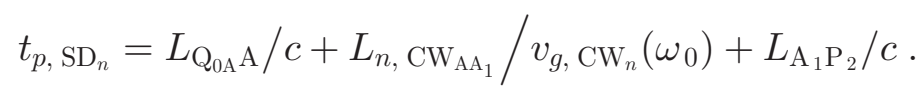

Here the group velocity of the transient $\mathrm{CW}_{n}$ element along the path $\mathrm{A} \curvearrowright \mathrm{A}_{1}\left(\equiv L_{\mathrm{CW}_{n}}\right)$ is defined by

$$
\begin{gathered}
v_{g, \mathrm{CW}_{n}}\left(\omega_{0}\right)=\frac{v_{p, \mathrm{CW}_{n}}\left(\omega_{0}\right)}{1-\omega_{0} v_{p, \mathrm{CW}_{n}}^{\prime}\left(\omega_{0}\right) / v_{p, \mathrm{CW}_{n}}\left(\omega_{0}\right)}(<c), \\
v_{p, \mathrm{CW}_{n}}^{\prime}\left(\omega_{0}\right)(<0) .
\end{gathered}
$$

Next, we consider the asymptotic analysis of the transient $\mathrm{CW}_{n} \mathrm{ED}$. The integral for $y_{\mathrm{CW}_{n} \mathrm{ED}}(\omega)$ obtained by substituting (6) into (11) is similar to the one for $y_{\mathrm{SD}_{n}}(t)$ in $(23)$.

By substituting the transformations for

$$
A_{\mathrm{SD}_{n}}(\omega) \rightarrow A_{\mathrm{CW}_{n} \mathrm{ED}}(\omega), \quad L_{n, \mathrm{CW}_{\mathrm{AA}_{1}}} \rightarrow L_{n, \mathrm{CW}_{\mathrm{AB}}}, \quad L_{\mathrm{A}_{1} \mathrm{P}_{2}} \rightarrow L_{\mathrm{BP}_{2}},
$$

into the asymptotic solution for $y_{\mathrm{SD}_{n}}(t)$ in (27), one may obtain the TD asymptotic solution for the transient $\mathrm{CW}_{n} \mathrm{ED}$ :

$$
\begin{aligned}
& y_{\mathrm{CW}_{n} \mathrm{ED}}(t) \sim A_{\mathrm{CW}_{n} \mathrm{ED}}\left(\omega_{0}\right) s_{0}\left(t-t_{p, \mathrm{CW}_{n} \mathrm{ED}}\right) \\
& \cdot \exp \left[-i \omega_{0}\left(t-t_{0}-L_{\mathrm{Q}_{0 \mathrm{~A}} \mathrm{~A}} / c-L_{n, \mathrm{CW}_{\mathrm{AB}}} / v_{p, \mathrm{CW}_{n}}\left(\omega_{0}\right)-L_{\mathrm{BP}_{2}} / c\right)\right] . \\
& t_{p, \mathrm{CW}_{n} \mathrm{ED}}=L_{\mathrm{Q}_{0 \mathrm{~A}} \mathrm{~A}} / c+L_{n, \mathrm{CW}_{\mathrm{AB}}} / v_{g, \mathrm{CW}_{n}}\left(\omega_{0}\right) v_{g, \mathrm{CW}_{n}}\left(\omega_{0}\right)+L_{\mathrm{BP}_{2}} / c .
\end{aligned}
$$

\subsection{TD asymptotic solutions for transient $\mathbf{R G O}_{k}$ and transient RED $_{k}$}

Substituting $u_{\mathrm{RGO}_{k}}(\omega)$ in (8) into the inverse Fourier transform $y_{\mathrm{RGO}_{k}}(t)$ in (11) yields the integral representation for the transient $\mathrm{RGO}_{k}$.

$$
\begin{gathered}
y_{\mathrm{RGO}_{k}}(t)=\frac{\exp \left(-\omega_{0} t_{0}\right)}{2 \pi} \int_{-\infty}^{\infty} A_{\mathrm{RGO}_{k}}(\omega) S_{0}\left(\omega-\omega_{0}\right) \exp \left[-i\left\{\omega t-h_{\mathrm{RGO}_{k}}(\omega)\right\}\right] d \omega \\
h_{\mathrm{RGO}_{k}}(\omega)=\omega\left(L_{\mathrm{Q}_{0 \mathrm{~A}} \mathrm{~A}} / c+L_{\mathrm{RGO}_{k}} / c\right) .
\end{gathered}
$$

The integral $y_{\mathrm{RGO}_{k}}(t)$ in (33) is described by the representation similar to the one $y_{m, \mathrm{ED}}(t)$ in $(15)$.

By substituting the transformations for

$$
A_{m, \mathrm{ED}}(\omega) \rightarrow A_{\mathrm{RGO}_{k}}(\omega), \quad L_{\mathrm{BP}_{1}} \rightarrow L_{\mathrm{RGO}_{k}}, \quad L_{m, \mathrm{ED}}(\omega) \rightarrow 0,
$$

into the integral $y_{m, \mathrm{ED}}(t)$ in (15) associated with (16), and then applying the Fourier transform method in Section 3.1, one may obtain the TD asymptotic solution for the transient $\mathrm{RGO}_{k}$ :

$$
\begin{gathered}
y_{\mathrm{RGO}_{k}}(t) \sim A_{\mathrm{RGO}_{k}}\left(\omega_{0}\right) s_{0}\left(t-t_{p, \mathrm{RGO}_{k}}\right) \exp \left[-i \omega_{0}\left(t-t_{0}-t_{p, \mathrm{RGO}_{k}}\right)\right], \\
t_{p, \mathrm{RGO}_{k}}=L_{\mathrm{Q}_{0 \mathrm{~A}} \mathrm{~A}} / c+L_{\mathrm{RGO}_{k}} / c .
\end{gathered}
$$

The TD asymptotic solution of the integral representation for the transient $\mathrm{RED}_{k}$ obtained by substituting (9) into (11) can be derived with the same procedure as the transient $\mathrm{RGO}_{k}$, and is given as follows:

$$
y_{\mathrm{RED}_{k}}(t) \sim A_{\mathrm{RED}_{k}}\left(\omega_{0}\right) s_{0}\left(t-t_{p, \mathrm{RED}_{k}}\right) \exp \left[-i \omega_{0}\left(t-t_{0}-t_{p, \mathrm{RED}_{k}}\right)\right],
$$




$$
t_{p, \operatorname{RED} k}=L_{\mathrm{Q}_{0 \mathrm{~A}} \mathrm{~A}} / c+L_{\mathrm{RED}_{k}} / c .
$$

\section{Numerical results and comparison with experimental results}

In this section we perform the numerical calculations and carry out the experiment to assess the validity of the novel TD asymptotic solution derived in Section 3 and to interpret the transient scattering phenomena when the $\mathrm{H}$-type plane pulse wave is incident on the edges of a cylindrically curved conducting open sheet.

We have shown in Fig. 2 (a) the response waveform vs. time curves observed at the point $\mathrm{P}\left(r, \psi=\psi_{\mathrm{ED}_{\mathrm{B}}}\right)$ on the tangent $\mathrm{ES}_{\mathrm{B}}$ (see Figs. 3 (a) and 3 (b)). Numerical parameters used in the calculations are given in the caption of Fig. 2 and the time $t$ is set $t=0$ when the plane pulse wave traverses the $y$-axis. The reference solution (- - : red dashed curve) has been calculated from the method of moment (MoM) and the fast Fourier transform (FFT) numerical code. The TD asymptotic solution (— : solid curve) has been calculated from a superposition of the transient scattered field in (10) when the pulse wave is incident on the edge A (see Fig. 3 (a)) and the one when the pulse wave is incident on the edge B (see Fig. 3 (b)). It is confirmed that the TD asymptotic solution ( - ) agrees excellently with the reference solution (- - -).

It is shown in Figs. 3 (a) and 3 (b) that 12 kinds of pulse elements (1) (12) arrive at the point P. From the arrival time differences of the transient pulse elements, the response waveform calculated from the reference solution can be divided into 4 wave packets (i), (ii), (iii), and (iv) (see Fig. 2 (a)). However, it is difficult to understand that 4 wave packets are composed of these 12 pulse elements. Therefore, in order to interpret in detail the transient scattered field and in order to assess the accuracy of the TD asymptotic solution for each pulse element which forms the response waveform, we have performed in Fig. 2 (b) the numerical calculation $(\bullet \bullet \bullet$ : closed circles) by using the FD asymptotic solution for the scattered field and the FFT numerical code. The closed circles $(\bullet \bullet \bullet)$ agree excellently with the reference solution $(---)$, so we use the numerical solution $(\bullet \bullet \bullet)$ as a new reference solution from now on.

Fig. 2 (c) shows the waveforms and the envelope shapes of pulse elements $(\mathbf{1} \sim 12)$. Each pulse element $(-)$ calculated from the TD asymptotic solution agrees excellently with the new reference solution $(\bullet \bullet \bullet)$ in every case. Please note that in order to avoid the confusion in Fig. 2 (c), only the waveform of each pulse element calculated from the new reference solution is indicated by the symbols $(\bullet \bullet \bullet)$. By comparing Fig. 2 (a) with Fig. 2 (c) and Figs. 3 (a) and 3 (b), we can easily recognize that the wave packets (i) and (ii) are identified as the $\mathrm{RGO}_{k=K_{1}=0}$ in 10 excited by the edge $\mathrm{B}$ (see Fig. 3 (b)) and the $\mathrm{RGO}_{k=0}$ in $\mathbf{4}$ excited by the edge A (see Fig. 3 (a)), respectively. While we can confirm that the bundle of pulse (iii) is made up of 6 pulse elements excited by the edge A, i.e., the $\mathrm{RGO}_{k=K_{1}=1}$ in (5), the $\mathrm{RED}_{k=0}$ in $\mathbf{6}$, the $\mathrm{RED}_{k=K_{2}=1}$ in $\mathbf{7}$, the $\mathrm{SD}_{n=N_{1}=1}$ in $\mathbf{3}$, and the GO and the ED elements in $(1)$ and $(2)$ which form the 


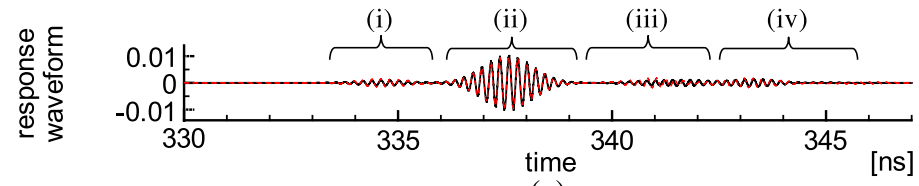

(a)

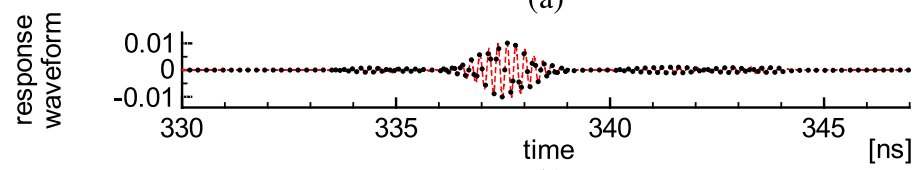

(b)

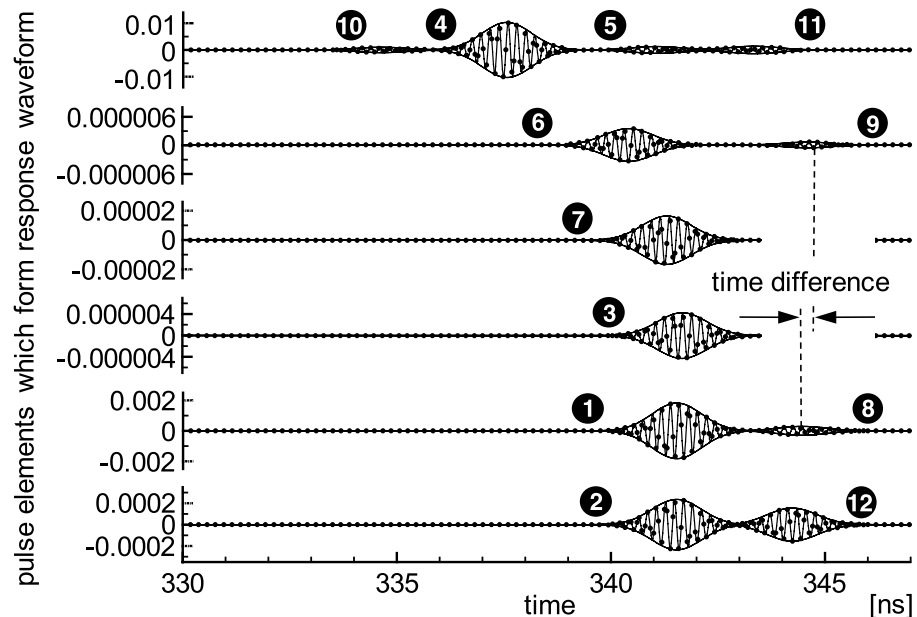

(c)

Fig. 2. Response waveform (Figs. 2 (a) and $2(\mathrm{~b})$ ) and pulse elements (Fig. 2. (c)) observed at the point $\mathrm{P}$ on the tangent $\mathrm{ES}_{\mathrm{B}}$. Numerical parameters: cylindrically curved conducting open sheet: $\left(a, \phi_{\mathrm{AB}}\right)=\left(1.0 \mathrm{~m}, 120.0^{\circ}\right)$ and observation point: $\mathrm{P}\left(r, \psi=\psi_{\mathrm{ES}_{\mathrm{B}}}\right)=\left(100.0 \mathrm{~m}, 30.3^{\circ}\right)$. Pulse source $s(t)$ in $(12)$ with $s_{0}(t)=\exp \left[-\left(t-t_{0}\right)^{2} /\left(4 d^{2}\right)\right]$ used in the calculation $\left(t_{0}=2.3 \times 10^{-9}[\mathrm{~s}], d=\right.$ $\left.4.1 \times 10^{-10}[\mathrm{~s}], \omega_{0}=3.0 \times 10^{10}[\mathrm{rad} / \mathrm{s}]\right)$. - - : reference solution, $\_$: TD asymptotic solution, new reference solution.

of 4 pulse elements excited by the edge B, namely, the $\operatorname{RED}_{k=0}$ in $\mathbb{1 1}$, the $\mathrm{CW}_{n=N_{2}=1} \mathrm{ED}$ in $\mathbf{9}$, the ED element in $\mathbf{8}$ which forms the $\mathrm{WG}_{m=M=1}$, and the $\operatorname{RED}_{k=K_{2}=1}$ in 12 (see Fig. $3(\mathrm{~b})$ ).

It is observed from Fig. 2 (c) that the $\mathrm{CW}_{n=N_{2}=1} \mathrm{ED}$ in 9 along the path $\mathrm{Q}_{0 \mathrm{~B}} \rightarrow \mathrm{B} \curvearrowright \mathrm{A} \rightarrow \mathrm{P}$ arrives at the point $\mathrm{P}$ later than the $\mathrm{ED}$ element in 8 along the path $\mathrm{Q}_{0 \mathrm{~B}} \rightarrow \mathrm{B} \rightarrow \mathrm{Q}_{1} \cup \mathrm{Q}_{2} \rightarrow \mathrm{A} \rightarrow \mathrm{P}$ (see Fig. $3(\mathrm{~b})$ ). From the path difference between the $\mathrm{CW}_{n=N_{2}=1} \mathrm{ED}$ and the ED and from the difference between the group velocities $v_{g}, \mathrm{CW}_{n=1}\left(\omega_{0}\right)(=0.994 c)$ in $(29)$ and $v_{g, m=1 \text {, ED }}(=0.981 c)$ in $(22)$, it is confirmed the interesting phenomena that the lowest-order $(n=1) \mathrm{CW}$ propagates along the curved conducting sheet from the edge $\mathrm{B}$ to the other edge $\mathrm{A}$ later than the lowest-order $(m=1)$ whispering-gallery mode.

We have obtained experimentally and numerically the transient scattered field by a cylindrically curved conducting open sheet. We have acquired the experimental data for the FD scattered field in the anechoic chamber by using a bistatic experimental setup. The measurements have 


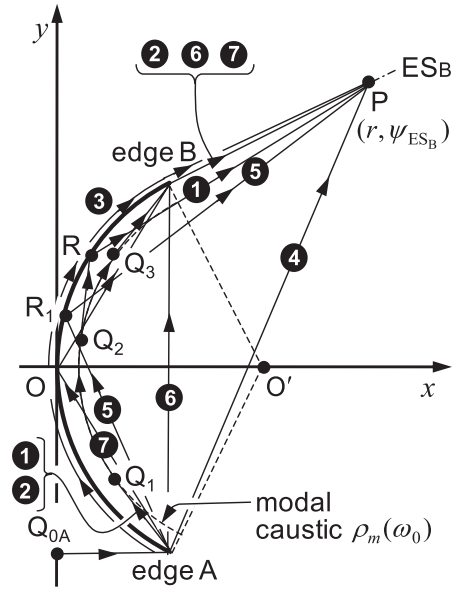

(a) Propagation paths of transient scattered field elements excited by the edge A.

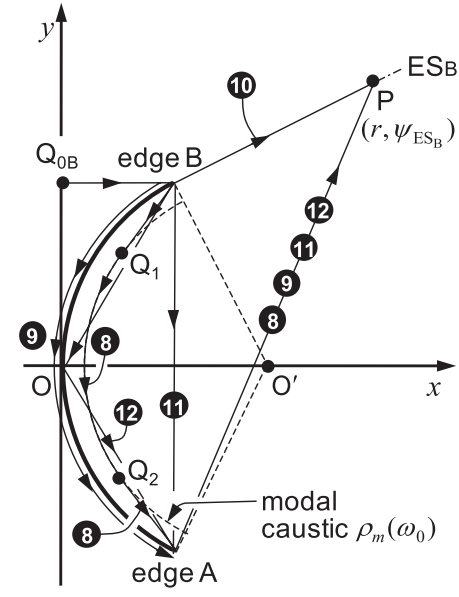

(b) Propagation paths of transient scattered field elements excited by the edge $\mathrm{B}$.

Fig. 3. Propagation phenomena of transient scattered field observed at the point $\mathrm{P}\left(r, \psi=\psi_{\mathrm{ES}_{\mathrm{B}}}\right)$ on the tangent $\mathrm{ES}_{\mathrm{B}}$ after excited by the edges $\mathrm{A}$ and $\mathrm{B}$.

been performed by changing only the transmitting frequency $f(=\omega /(2 \pi))$ while keeping the curved conducting sheet, the transmitting antenna, and the receiving antenna. Then we have calculated numerically the transient scattered filed by using the FD experimental data and the FFT numerical code. We use the results obtained experimentally and numerically as the experimental-numerical results.

We have shown in Fig. 4 the comparison of the TD asymptotic solution (— : solid curve) with both the experimental-numerical results $(\bullet \bullet \bullet$ : green closed circles) (Fig. 4 (a)) and the reference solution (- - -: red dashed curve) (Fig. 4 (b)). It is observed that the TD asymptotic solution ( - ) agrees excellently with both the experimental-numerical results $(\bullet \bullet \bullet)$ and

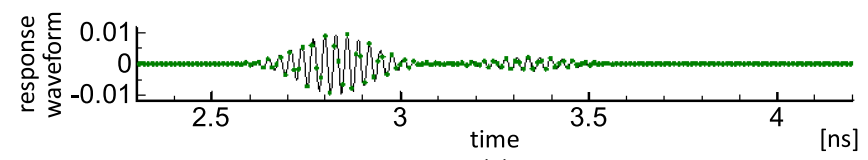

(a)

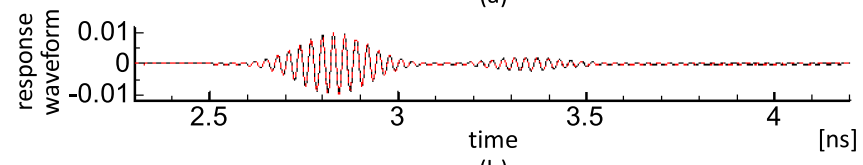

(b)

Fig. 4. Comparison of TD asymptotic solution with both the experimental-numerical results (Fig. 4 (a)) and the reference solution (Fig. 4 (b)). Numerical parameters: cylindrically curved conducting open sheet: $\left(a, \phi_{\mathrm{AB}}\right)=\left(1.01 \times 10^{-1} \mathrm{~m}, 99.6^{\circ}\right)$ and observation point: $\mathrm{P}(r, \psi)=\left(0.8 \mathrm{~m}, 80.4^{\circ}\right)$. Pulse source $s(t)$ in (12) with $s_{0}(t)=\exp \left[-\left(t-t_{0}\right)^{2} /\left(4 d^{2}\right)\right]$ used in the calculation $\left(t_{0}=3.14 \times 10^{-10}[\mathrm{~s}], d=6.6 \times 10^{-11}[\mathrm{~s}]\right.$, $\left.\omega_{0}=2.1 \times 10^{11}[\mathrm{rad} / \mathrm{s}]\right)$. — : TD asymptotic solution, $\bullet \bullet$ : experimental-numerical results, - - reference solution. 
the reference solution (- - ).

From Figs. 2 and 4, the validity of the novel TD asymptotic solution presented here is confirmed both theoretically and experimentally.

\section{Conclusion}

We have derived the novel time-domain (TD) asymptotic solution for the transient scattered field excited by the edges of a cylindrically curved conducting open sheet by applying only the Fourier transform method. By comparing with both the reference solution and the experimental-numerical results, we have confirmed the validity of the TD asymptotic solution which is represented by a superposition of the transient scattered field elements arrived at the observation point. We have confirmed the interesting phenomenon that the creeping wave propagates along the curved conducting sheet later than the whispering-gallery mode.

\section{Acknowledgement}

This work was supported in part by the Grant-in-Aid for Scientific Research (C) (24560492) from Japan Society for the Promotion of Science (JSPS). 